\title{
Osteoarthritis and fear
}

\begin{abstract}
Osteoarthritis, a largely intractable health condition with incalculable health and financial and social costs and ramifications among many older adults remains an essentially intractable condition. Commonly instructed to exercise to combat pain, the role of fear is seldom discussed as an enormously influential exercise impediment in this regard. This mini review highlights aspects of the research that imply consideration of fears rather than exercise prescription alone may be more helpful than not in efforts to allay the enormous suffering experienced by most older adults diagnosed as having osteoarthritis of one or more joints.
\end{abstract}

Keywords: avoidance behavior, anxiety, disability, fear, intervention, osteoarthritis, pain, treatment
Volume 6 Issue 4 - 202I

\author{
Ray Marks \\ Department of Health and Behaviour Studies, Teachers College, \\ Columbia University, USA
}

\begin{abstract}
Correspondence: Dr. Ray Marks, Department of Health and Behavior Studies, Teachers College, Box I I4, 525W I20th Street, New York, NY 10027, USA, Tel I-212-678-3445, Fax I-2| 2-678-8259, Email rm226@columbia.edu
\end{abstract}

Received: November 27, 2021 | Published: December 10, 202

\section{Overview}

Osteoarthritis, a widespread largely degenerative disease of one more freely moving joints, such as the hip, knee or hand joints, is the most common crippling disabler of older adults. ${ }^{1,2}$ Associated with multiple pathological changes in the tissues comprising and surrounding the affected joint, the disease is also associated with immense bouts of pain, various degrees of swelling around the affected joint, muscle weakness, and often joint instability. The disease, which is largely progressive, and not readily amenable to most known treatment approaches, ${ }^{1}$ consequently commonly produces multiple challenges to mobility, the ability to function physically, economically, and socially, as well as independently. Commonly accompanied by high levels of emotional distress, depression and/or impairments of general health and vitality, reduced life quality, activity limitations, negative personal beliefs, as well as sleep disturbances, the older adult suffering from osteoarthritis may have multiple fears and anxieties that can hamper their ability to adapt successfully to their disease. These changes and others such as poor cardiovascular endurance, low exercise tolerance, sleep challenges, and a diminished ability to function physically without pain and undue exertion can also increase the risk or exacerbate other health problems such as obesity, frailty, and a proclivity for falling. At present however, and despite years of study, very little attention is given to the interacting physical attributes of osteoarthritis and the psychological correlate of fear, even though there is no argument that the burden of the disease is multi dimensional in origin and is immense. ${ }^{3,4}$

In particular, even though many diverse factors associated with osteoarthritis can undoubtedly provoke various fears, such as unremitting stress, falls injuries, comorbid health conditions, and fear of drug dependency, and fear is a clear cognitive attribute in its own right as opposed to anxiety ${ }^{5}$ to which it can lead, more attention to anxiety is clearly given as regards its association with pain, movement and osteoarthritis disease progression discussions. In addition, fear, a cognitive attribute that is quite well studied in other spheres, such as cardiovascular disease, where it is found to commonly generate both specific as well as a distinctive set of behaviors, as well as having distinctive antecedents that separate it from anxiety and panic, ${ }^{5}$ is only marginally mentioned and poorly studied in the context of the entire osteoarthritis literature. This is despite its implications for advancing more insightful disease understandings, understanding why some sufferers may reject surgery, or invasive tests or both, or fail to demonstrate degree of progress anticipated in response to therapy.
Consequently, how fear can evolve as well as involve both acute as well as maladaptive threat responses in this condition, the adoption of which may be highly disadvantageous in the long term to overall joint health as well as well being is poorly understood at best. ${ }^{6}$

\section{Objective}

In light of the possible importance of the issues mentioned above, this current review sought to examine what is known about this fear topic insofar as having implications for clinical providers, researchers, as well as patients in terms of efforts to heighten disease understandings as well as the possibly of averting undue suffering attributable to osteoarthritis. Specifically sought was information on osteoarthritis as this occurs at multiple joint sites other than the knee and hip, such as the neck, low back, hands, feet, ankles, shoulders, wrist, elbow and jaw among the older population, as well as the possible destructive role of various disease associated fears.

\section{Hypothesis}

It was hypothesized that the emotion of fear and its possible association with accompanying symptoms of osteoarthritis other than pain, such as anxiety, depression, muscle weakness, poor proprioception and balance capacity, low pain, coping, and functional self-efficacy and self-image, daily living challenges, and work disability would be an attribute warranting consideration by clinicians.

\section{Relevance}

The key reason for focusing on the attribute of fear in this review was its possible potentially unrecognized strong influence on joint status and especially on overall osteoarthritis disease progression rates, and extent, plus overall social outcomes and functional disability, hence opening the door to more insightful disease prevention and remediation approaches.

\section{Rationale}

Osteoarthritis, the most prevalent chronic disease, ${ }^{7}$ involves the disruption of many joint tissues as well as painful inflammatory processes. Involving multiple biomechanical abnormalities and challenges that impact function, ${ }^{8}$ it is possible however, to limit these interactive destructive processes to some degree, through both nonpharmacologic as well as pharmacologic approaches. However, the presence of cognitive factors that may prevail in the forms of trait anxiety, for example, plus those emerge over time, such as various 
fears, and that can be shown to impact joint destructive processes indirectly, are seldom addressed either in the context of efforts to better understand the disease, and especially in its treatment and management. As a result, it can be conjectured that many cases affected by this irreversible chronic disease than not will and do suffer excess disability, while continuing to incur unrelenting fears that may not only drive unhealthful behaviors and cognitions, but stress levels that predictably impact health and pain significantly and negatively. This is unfortunate because pain cognitions, particularly pain catastrophizing-or exaggerated pain responses due to excess fears, appear to be important variables in the context of efforts to understand why some cases affected by osteoarthritis are more disabled than others. The variable of fear may also provide insight into why most intervention approaches fall short and may fail to either provide optimal results, or sufficient adherence levels, even if the pathology is comparable. ${ }^{9}$ In particular, exercise, the most commonly recommended antidote against osteoarthritis may not be pursued as outlined due to fears of pain, muscle weakness, and possible instability..$^{10}$ As well, unaddressed social fears may be expected to further preempt the use of unsightly albeit recommended braces or joint supports, orthopedic shoes/footwear, walking or mobility aids, as well as some form of dependency in general, resulting not only in greater exposure to mechanical forms of injury and pain, but greater feelings of distress and possible anxiety. In addition, in the case of the overweight or obese osteoarthritis sufferer who is especially likely to experience pain with movement, this sub group may arguably be more likely to develop pain-related fears of movement that in turn may serve as obstacles or barriers to both weight control as well as involvement in daily activities or exercise regimens that would otherwise help them to control both their weight, as well as pain. ${ }^{11}$ The use of the internet to foster rehabilitation among older adults with various degrees of osteoarthritis who may be especially fearful to move in the absence of personal professional help and oversight may also account in part for null results or suboptimal and possibly harmful outcomes, rather than lack of motivation reported by Mandl et al., ${ }^{12}$ if the psychological correlate of fear is not addressed. ${ }^{13}$ Fear as a correlate including the fear of physiotherapy exercises, as well should not be overlooked or remain unaddressed. Moreover, simply encouraging exercise even though it may be injurious to an unstable or inflamed joint could not only evoke pain, but fear of movement, plus the potential for worse outcomes, rather than for disease amelioration, a goal highlighted more than a decade ago. ${ }^{14}$

Unfortunately, as recounted by Heuts et al., ${ }^{15}$ although fear of pain and disability, as well as inflammation are closely related osteoarthritis factors, existing osteoarthritis literature, as well as care models tend to focus primarily on examining osteo arthritic joint tissue radiographic and biological pathways in efforts to either diagnose the condition and/or to solve the osteoarthritis pathological puzzle. ${ }^{16}$ As well, this limited viewpoint along with a variety of palliative biomedical or antiinflammatory interventions, such as corticosteroid injections, antiinflammatory drug usage, opioid use, or joint exploratory surgeries and intra-articular therapies based on these studies are not commonly optimally effective, and are all likely to be impacted negatively where fear prevails. ${ }^{2}$ This also applies to total joint replacement surgery, an intervention that may not directly impact upon possible mediating or moderating cognitive factors, such as fear, that can arguably induce excess rates of joint degeneration and inflammation, high levels of sedentary activities, muscle weakness, and joint instability. ${ }^{17}$

\section{Methods}

To assist in achieving the aims of this mini review, the PUBMED, PUBMED CENTRAL, PSYCINFO, SCOPUS, ACADEMIC
SEARCH COMPLETE, and GOOGLE SCHOLAR depositaries believed to house salient topical peer reviewed articles were employed. Key terms used included, osteoarthritis and fear, osteoarthritis disability and fear. After an extensive search, all pertinent data addressing the current topic were downloaded and those deemed of substantive relevance are presented in this report solely in a narrative form, given the low number of topical papers, or any well controlled studies or studies in general on this topic. Excluded were articles on rheumatoid arthritis, knee surgery conditions other than osteoarthritis, protocols for intervention, and surveys being developed to assess fear associated emotions.

\section{Results}

Very limited numbers of papers were found to prevail on this topic, for example on PSYINFO only 46 articles are listed, regardless of year, and among more than 8000 papers on osteoarthritis published on PUBMED in 2020-2021, only 40 were inclusive of the keyword fear. Among these, moreover, other than discussions on fears of falling and pain, very few other forms of fear were mentioned. As well, almost no report could be found that mentioned osteoarthritis as this occurs at multiple joints, other than the knee or hip. Those mentioning possible methods of ameliorating fear symptoms using exercises, for example exercise participation to overcome the fear of falling, surprisingly showed no remarkable impact. ${ }^{18}$ However, clearly, some cases with a diagnosis of osteoarthritis in the study by Mat et al. ${ }^{18}$ may not have improved sufficiently due to their fear of movement ${ }^{17}$ or weakness ${ }^{10}$ or both, which may conceptually be linked in turn to depression as well as limited physical activity participation, ${ }^{19}$ potential pathogenic attributes not truly addressed. Other subjects may have had excessive pain due to their fears of medication dependency, and/or misinterpretation of health messages, ${ }^{20}$ and various intervention fears that were not addressed, and thus were both in too much pain, as well as reluctant to actively exercise. As outlined ${ }^{17}$ fear of movement among cases with symptomatic knee osteoarthritis may be especially high, because this joint is only often very painful, but is often unstable, and may buckle or 'lock' readily on weight bearing. Other research implies that in this instance or others like this, where fear is then evoked, but is not adequately acknowledged or addressed, this could conceivably impact future levels of physical activity participation or willingness to participate in actions that would possibly prove beneficial otherwise. ${ }^{19}$ Kopp ${ }^{9}$ who examined several psychological attributes of osteoarthritis cases found their model for pain intensity to include time spent exercising and fear of painful movement.

In another study designed to examine whether fear of movement, depression and functional performance are predictors of physical activity level in cases with knee osteoarthritis, Kilinç et al. ${ }^{21}$ who studied 200 cases concluded it is important to improve physical activity levels in this group early on before the disease progresses and becomes more costly to treat. But the actual cause of the fear and what should be done was unclear, although Selcuk et al. ${ }^{19}$ mention that simply focusing on pain relief is probably insufficient.

The possible presence of fear does however appear important to address given that fear of movement is found to have a negative impact not only on physical function, aerobic fitness, self-efficacy, and disability per se, ${ }^{22}$ but on outcomes following total knee joint arthroplasty surgery, ${ }^{23}$ as well as total hip joint arthroplasty. ${ }^{24}$ Fear may also greatly impact stair descent ability in cases of knee osteoarthritis, ${ }^{25}$ as well as the patient's belief that they can function physically and that often leads to disability. ${ }^{22}$ Persistent fear states have also been observed to directly impact brain neural patterns in efforts of the individual to cope with fear related pain-anxiety generated by 
osteoarthritis, ${ }^{26}$ thus these maladaptive approaches should be thwarted early on in order to safeguard against emergent resistant anxiety states. ${ }^{27}$ As observed by Kocic et al..$^{28}$ pain hypervigilance leading to excess fear of movement ${ }^{29}$ can perpetuate pain, joint flexion and functional deficits even after reconstructive joint replacement surgery, designed to reduce pain and these deficits. In addition, research shows that these movement associated fears commonly exist pre-operatively, and if not addressed, do tend to predict unwanted postoperative functional limitations, despite any technically successful surgery. Moreover, this attribute of kinesiophobia is more common than not among post surgical joint replacement patients where it does appear associated with the likelihood of a low rather than a high life quality. ${ }^{24}$

Thus even though ${ }^{30}$ concluded that, fear of movement, selfefficacy beliefs and fear of falling associated with levels of disability in people with osteoarthritis of the knee are not of high import, because falls do not appear to heighten disability levels to the extent that pain and stiffness do, this is not supported by other research findings in this sphere. Indeed, several clearly show it is possible that cases who experience fears of moving as well as fears of injury ${ }^{31}$ or re injury, ${ }^{32}$ as well as those who fear physiotherapy, or falling after total knee arthroplasty surgery are quite numerous, especially among women and those with high anxiety or body mass, ${ }^{33}$ who will possibly experience more pain than desirable as a result. ${ }^{34}$ They are also likely to be more predisposed to increases in joint derangement and disability as shown by Helminen et al. ${ }^{35}$ where fear of movement predicted worse functioning and as confirmed by Odele et al. ${ }^{36}$ Fear of movement also predicts numbers of steps walked each day in knee osteoarthritis cases, ${ }^{37}$ as well as self efficacy for function, and fear of pain and activity participation. ${ }^{38}$

As outlined by Sánchez-Herán et al., ${ }^{39}$ as well as pain and stiffness, persons with knee osteoarthritis may not only be at risk for incurring unstable joint sensations and actual instability during weight bearing activities that often leads to a fear of movement, but together with movement avoidance, this may engender fear of injury and re injury, as well as actual functional impairments that exacerbate injury risk, ${ }^{40}$ falls risk, and increase the risk for frailty. ${ }^{41}$ Moreover, resultant impairments in physical functioning as well as periods of excess pain catastrophizing, and fear avoidance beliefs leading to limitations in many life affirming activities, such socializing, ${ }^{42}$ and participation in recreational activities, predict both excess aging disability, depression, heightened co morbid disease risks, and premature mortality. ${ }^{43}$ As well, Aydemir et al. ${ }^{11}$ who studied knee osteoarthritis cases, concluded that muscle strength declines that commonly accompany the disease may be further and predictably impacted due to the impact of movement fears that lead to adoption of sedentary behaviors. In turn, the onset of excess muscle weakness appears to influence the pursuit of physical activities both directly, as well as indirectly, as well as in the context of surgery. ${ }^{31,44}$

Hence, the possible presence and extent of those fears that foster activity avoidance, and which are frequently coupled to or lead to feelings of anxiety, anxiety sensitivity, depression, and harmful threat perceptions among older osteoarthritis sufferers ${ }^{28,45}$ are all potentially key moderators or mediators that can unexpectedly impact the success of any remedial osteoarthritis intervention effort, even if it is evidence based and well intentioned. In this regard, it appears efforts to foster adherence to the most common and life affirming osteoarthritis self-management recommendations, parallel efforts to reduce any excessive susceptibility to fears and their consequences are clearly desirable and to this end, several therapeutic approaches have been discussed. Among these, one of the most promising in efforts to reduce unrealistic patient fears of various osteoarthritis symptoms and ensuing impairments as this might impact their daily and social life, as well as life quality is that of Alami et al. ${ }^{46}$ of an appropriately adapted formalized education process, plus a comprehensive global assessment approach, and accurate drug therapy information. Another is that of Whetherell et al. ${ }^{47}$ involving an integration of exercise and exposure therapy plus verbal interventions that both target personal expectancies, as well as targeting maladaptive beliefs among osteoarthritis cases. ${ }^{28}$ Another is the self-management and exercise approach discussed by Jonsson et al. ${ }^{48}$ and that might include efforts to foster skills mastery, falls, pain coping, and self-efficacy/positive beliefs. ${ }^{49}$ Others that have been proposed and need to be examined empirically in well-designed trials include: aquatic exercises,${ }^{50}$ brain targeted treatments, ${ }^{51}$ joint protection/general education, ${ }^{20,24,52,53}$ approaches that increase physical activity, ${ }^{19,21,38}$ Tai Chi, ${ }^{54}$ and guided imagery, drawings, and education. ${ }^{24}$

Web based osteoarthritis interventions and others that aim to reduce fears $^{34,55}$ and that might consider proving information on medication safety, exercise safety, home modifications, and injury prevention may also prove highly practical and efficacious for selected cases.

\section{Discussion}

Osteoarthritis, the most common disease affecting the mobility and independence of many older adults and one generally resistant to most current modes of intervention, even when surgery is implemented, remains an enormous public health and human challenge in an aging global society. In this regard, emerging research indicates that the limited focus on psychological factors to date, as opposed to physical factors that can markedly and conceivably impact the osteoarthritis disease cycle quite negatively may partially explain this widespread less than desirable disease outcome despite years of research. In this introductory review we thus sought to specifically examine the strength of the evidence for more concerted clinical efforts to both assess as well as treat any persistent disease associated fears where present. We also sought to highlight any evidence that could provide a sound rationale for more efforts in remediation approaches to address fears early on in the disease process, as well as over the course of the disease. This was done in an effort to improve the scope of care that might greatly improve the outlook for many older adults suffering from chronic disabling osteoarthritis of one or more joints. It was hoped this endeavor would reveal whether more could be done in the clinical realm to offset both fears of a diverse nature and their potential for enhancing negative outcomes despite well-intentioned health recommendations.

As per Lentz et al. ${ }^{56}$ and Rosemann et al..$^{53}$ it is increasingly evident that not only is this a complex disease involving multiple pain producing biochemical and biomechanical features not readily reversible or amenable to intervention, but current biomedical interventions clearly commonly fail to address the multiple and diverse psychological correlates known to influence osteoarthritis disability, pain and joint pathology. This is surprising because in terms of relationships among the variables analyzed presently, there were several noteworthy bivariate correlations between structural disabling problems and cognitions such as excess fear. Indeed, this specific topic, while of considerable import, because movement avoidance in its own right is a pathological predictor of the disease process, is surprisingly less well articulated in this respect when compared to depression and anxiety, or pain, with which it is related. Also most research focuses solely on one dimension of fear, even though this term includes, but is not limited to fears of pain, movement and reinjury, as well as fears of disability, fears of medication, dependency, surgery, and fears of falling and others. ${ }^{57}$ As well Ikram et al..$^{58}$ noted 
the most common fear factor according to patients priority for total knee replacement therapy was worsening of symptoms after total knee replacement $42(40 \%)$ followed by pain persistence after total knee replacement $27(25.7 \%)$, financial feasibility $21(20 \%)$, and religious problems $15(14.3 \%)$. Since unresolved fear is an emotion linked to greater levels of pain and disability, exaggerated pain levels, persistent pain, deconditioning, physical disuse ${ }^{57}$ and high dissatisfaction with joint replacement surgery, where it is in turn linked to fear avoidance, ${ }^{59}$ failing to account for this potentially potent disease mediator or moderator both in the clinic as well as the hospital before or after surgery appears short sighted at best.

Indeed, increasing data, although not all ${ }^{60}$ support the idea that providers need to develop more skill at both anticipating possible perceptual vulnerabilities such as pain fears, as well as at addressing and uncovering the origins of this possible attribute of excess suffering among older adults with osteoarthritis of one or more joints. This could include the ability to empathetically discuss the nature of their disease with them, plus concerted efforts to offer tailored services directed towards improving their pain coping skills, and mitigating physical stresses, as well as mental stress associated factors such as anger, frustration, discouragement fear and helplessness, ${ }^{61}$ as well as fears of partaking in physical therapy or pain producing activities..$^{55}$ In addition monitoring pain during and after exercise, providing days of rest when disease flares occur, and infusing variety into the exercise regimen are recommended to encourage adherence. ${ }^{62}$

The role of relaxation training and the enhancement of executive functioning skills and emotional regulation, ${ }^{63}$ advocating for psychological therapies as indicated, in addition to physical therapies, along with education, especially among those in high distress or those cases with obvious negative pain coping strategies and/or unstable unprotected joints also appears highly warranted.

At the same time, and to advance this field of endeavor, in addition to embracing considerations of the ongoing or prevailing presence or possible emergence of excess fear in their assessments and advisories, providers may help to direct their 'at risk' osteoarthritis older clients to resources that can provide psychotherapy as well as physical therapy, as indicated, especially where this can clearly help foster safe but desirable bouts of physical activity ${ }^{64}$ as well as avoiding stressful joint movements. In addition, future studies to evaluate whether more careful tailoring of treatment recommendations to accommodate specific osteoarthritis psychological phenotypes will yield more favorable clinical outcomes than non tailored treatments, or treatments with a more biomedical orientation are likely to prove valuable. In the meantime, it appears safe to conclude that the presence of high levels of intractable pain coupled with muscle weakness and joint instability, will potentially foster multiple pain-related fears that in turn, will greatly impact functional ability and independence, as well as feelings of depression and poor life quality ${ }^{64}$ if untended or recognized, even in cases with minimal radiological damage. ${ }^{15}$

As well, although Youngcharoen et al. ${ }^{60}$ found pain related fears of no import in influencing knee osteoarthritis health status, Skopaz et al. ${ }^{65}$ note both high anxiety and fear-avoidance beliefs to be related to poorer function, pain and osteoarthritis disability. As well fear of falling, balance, and physical capacity in the case of knee osteoarthritis appears to be significantly related. ${ }^{66}$

In short, even though the role of fear or fear factors in general is challenging to delineate and seldom discussed in the recent osteoarthritis literature, the presence of fear is a symptom commonly expressed as a highly important concern by patients, ${ }^{67}$ including possible fears of injury, disfigurement, social fears, fears of cardiac symptoms in those with comorbid health conditions, work and financial fears and others, and pain, which may all have a bearing on the extent of osteoarthritis suffering and pathogenic manifestations. Unfortunately, regardless of data base employed, not only do current articles describing osteoarthritis pathology or treatment mostly fail to mention fear in any form, for the most part, but those that do largely allude to fear factors in the context of knee osteoarthritis, where they discuss fears of pain and movement as well as falling, but fail to discuss other joints, cases with multiple joint sites affected, and how fear might manifest in other ways and correlate with its molecular pathology, and overall patient wellbeing. As well, leading organizations overseeing osteoarthritis care recommendations currently fail to endorse or even mention its possible importance. . $^{1,2,14,16,68}$

However, based on what is reported, as well as knowledge of osteoarthritis pathology, it seems reasonably safe to assert with modest confidence that the emotion of fear, which is likely to prevail among many older adults both as a comorbid condition in its own right, is likely to prevail among many older adults suffering from excess pain. Adding to these fears is the widespread myth that 'nothing' can be done to mitigate this disease, ${ }^{46}$ and that providers rarely address emotional aspects of this disease, ${ }^{46,56}$ despite evidence that their patients may predictably suffer worse outcomes than not if one or more of their disease associated fears remains unabated, ignored or overlooked. ${ }^{71}$ Moreover, one can safely assume exercise recommended self-management regimens and others will be highly challenging to successfully pursue or adhere to in the presence of excess fear, and lack of confidence in carrying these activities regardless of whether these are to be applied in either a non surgical or a surgical situation. ${ }^{72}$ A pathway of destruction of the osteoarthritic joint implicating fear as a strong predictor that might negatively affect osteoarthritis functional and structural dimensions over time is outlined below in Figure 1, and warrants further research, especially qualitative combined with quantitative and molecular approaches in our view.

OSTEOARTHRITIS - pain, joint swelling, instability, --- fear movement/re injury/pain, anxiety + depression,
poor self-efficacy, avoidance behaviors obesity, metabolic syndrome, slow reflexes, walking speeds -abnormal joint
reactive forces, increased falls risk, fear falling, frailty, social impacts
Lower functional status, lower recovery status post surgery, inactivity, higher overall health risks. long-term
disability-frailty, repeated doctors visits, polypharmacology, surgery with persistent pain, post surgical
dissatisfaction, decreased life quality

Figure I Schematic representation of possible morbidity impact of untreated fear and poor psychological adjustment to fear in older cases of osteoarthritis. Key Sources [II, 22, 32, 4I, 42, 45, 62, 69, 70-75].

\section{Conclusion}

After extensive consideration and in light of the above discourse and available data we conclude:
Fears or fear factors, commonly accompanying the enormous challenges faced by older adults with painful disabling osteoarthritis of one or more joints can arguably impact the extent of osteoarthritis as well as comorbid debility and suffering quite markedly, even if 
not well researched or acknowledged in the mainstream literature and practical fields of endeavor. Moreover, it can safely be concluded that unrelieved or inadequately addressed fears along with treatments that are not only somewhat suboptimal but focus largely on the physical features of osteoarthritis, rather than behavioral and psychological attributes, is more likely than not to foster a more rapid, extensive, and severely disabling state of dysfunction, plus more rapid and extensive disease progression, pain sensitivity, heightened anxiety states, poor surgical outcomes and others, especially among the older adult population.

As well as possibly increasing the costs of care, plus the need for more extreme or invasive forms of treatment, a parallel contribution of unremitting fears and negative counter behaviors leading to possible heart strain, high blood pressure, and/or depression, plus obesity, increased falls risk, low self-efficacy, need for opioids, sleep disturbances, and the associated risk of frailty can be predicted to further hasten the rate and magnitude of any prevailing joint destructive processes, even in the face of most recommended management strategies and possible surgery, if ignored.

To this end, providers willing to shift or heighten their focus to encompass a more patient-centered and holistic personalized approach, rather than solely a pathological one, and one that deliberately take the time to listen to and to thoughtfully assess their clients comprehensibly at the outset as far as their personal osteoarthritis outcome beliefs, past practical experiences, educational as well as any pervasive health challenges, fears, doubts, and dreads, and falls history, are concerned, while providing an empathetic but optimistic outlook about the client's ability to overcome any unwarranted fears and worries they may have, will potentially enable the patient to assume more effective control of their lives, and thereby a more advantageous 'upward, rather than a downward spiral' of physical as well as mental health attributes of wellbeing. In addition, helping older adults with osteoarthritis to deal with, recognize, or eliminate any modifiable degree of unwarranted fear that is possibly maladaptive and not based on a possible actual threat, as well as extrinsically derived fear determinants, such as fears of physiotherapy, fears of falling, or fears due to social and environmental factors, which appears humanistic in its own right, is likely to greatly reduce the immense and oftentimes overwhelming less visible suffering of osteoarthritis, including its social and fiscal burden that alone can impede healing and recovery and foster physical illness.

At the same time, it is concluded that helping to ensure clients receive counseling as required, as well as helping the older adult with unremitting osteoarthritis to obtain appropriate informational, instrumental, and empathetic albeit skilled professional support in light of patients' preferences and situation will potentially help to foster more affirmative pain self-efficacy beliefs, functional ability, and overall self-confidence and is strongly encouraged regardless of whether the fears are physical or psychological in origin. Moreover, in addition to careful assessment, and one that engages the patient's trust and co-operation, reiterating the importance of recommended actions using credible evidence and providing empathetic support as indicated, may be expected to markedly facilitate the adoption and adherence of desirable osteoarthritis health recommendations, while minimizing dysfunctional outcomes, regardless of anticipated fears, and extent of disablement.

\section{References}

1. Sacitharan PK. Ageing and osteoarthritis. Subcell Biochem. 2019;91:123159.

2. Nelson AE. Osteoarthritis year in review: clinical. Osteoarthritis Cartilage. 2018;26(3):319-325
3. Litwic A, Edwards MH, Dennison EM, Cooper C. Epidemiology and burden of osteoarthritis. Br Med Bull. 2013;105:185-199.

4. Vina ER, Kwoh CK. Epidemiology of osteoarthritis:literature update. Curr Opin Rheumatol. 2018;30(2):160-167.

5. Perusini JN, Fanselow MS. Neurobehavioral perspectives on the distinction between fear and anxiety. Learn Mem. 2015;22(9):417-425.

6. Norrholm SD, Jovanovic T. Fear processing, psychophysiology, and PTSD. Harv Rev Psychiatry. 2018;26(3):129-141.

7. Bortoluzzi A, Furini F, Scirè CA. Osteoarthritis and its managementepidemiology, nutritional aspects and environmental factors. Autoimmunity Reviews. 2018;17(11):1097-1104.

8. Glyn-Jones S, Palmer AJ, Agricola R, et al. Osteoarthritis. The Lancet. 2015;386(9991):376-387.

9. Kopp B, Furlough K, Goldberg T, et al. Factors associated with pain intensity and magnitude of limitations among people with hip and knee arthritis. J Orthop. 2021;25:295-300.

10. Aydemir B, Huang CH, Foucher KC. Strength and physical activity in osteoarthritis: The mediating role of kinesiophobia. J Orthop Res. 2021.

11. Somers TJ, Keefe FJ, Pells JJ, et al. Pain catastrophizing and pain-related fear in osteoarthritis patients: relationships to pain and disability. $J$ Pain Symptom Manage. 2009;37(5):863-872.

12. Mandl LA. Osteoarthritis year in review: clinical. Osteoarthritis Cartilage. 2019;27(3):359-364.

13. Sharma L. Osteoarthritis of the knee. NEJM. 2021;384(1):51-59.

14. Bijlsma JW, Berenbaum F, Lafeber FP. Osteoarthritis: an update with relevance for clinical practice. Lancet. 2011;377(9783):2115-2126.

15. Heuts P, Vlaeyen J, Roelofs J, et al. Pain-related fear and daily functioning in patients with osteoarthritis, Pain. 2004;110(1):228-235.

16. Geyer M, Schönfeld C. Novel Insights into the pathogenesis of osteoarthritis. Curr Rheumatol Rev. 2018;14(2):98-107.

17. Gunn AH, Schwartz TA, Arbeeva LS, et al. Fear of movement and associated factors among adults with symptomatic knee osteoarthritis. Arthritis Care Res (Hoboken). 2017;69(12):1826-1833.

18. Mat $\mathrm{S}, \mathrm{Ng} \mathrm{CT}$, Tan PJ, et al. Effect of modified Otago exercises on postural balance, fear of falling, and fall risk in older fallers with knee osteoarthritis and impaired gait and balance: A secondary analysis. PM\&R. 2018;10(3):254-262.

19. Aykut Selçuk M, Karakoyun A. Is there a relationship between kinesiophobia and physical activity level in patients with knee osteoarthritis?. Pain Medicine. 2020;21(12):3458-3469.

20. Williams NH, Amoakwa E, Burton K, et al. The Hip and Knee Book: developing an active management booklet for hip and knee osteoarthritis. Br J Gen Pract. 2010;60(571):64-82.

21. Kilinç H, Karahan S, Atilla B, et al. Can fear of movement, depression and functional performance be a predictor of physical activity level in patients with knee osteoarthritis? Arch Rheumatol. 2018;34(3):274-280.

22. Bhatt NG, Sheth MS, Vyas NJ. Correlation of fear avoidance beliefs with pain and physical function in subjects with osteoarthritis of knee (OA knee). Int J Ther Rehabil Res. 2015;4:117-121.

23. Sullivan M, Tanzer M, Stanish W, et al. Psychological determinants of problematic outcomes following total knee arthroplasty. Pain. 2009;143(1):123-129.

24. Padovan AM, Kuvačić G, Gulotta F, et al. A new integrative approach to increase quality of life by reducing pain and fear of movement in patients undergoing total hip arthroplasty: The IARA model. Psychology, Health \& Medicine. 2018;23(10):1223-1230.

25. Stendotter A, Roeleveld K, Roeleveld K, et al. Factors associated with self-rated difficulty to descend stairs in persons with knee osteoarthritis. PM\&R. 2021. 
26. Keszthelyi D, Aziz Q, Ruffle JK, et al. Delineation between different components of chronic pain using dimension reduction - an ASL fMRI study in hand osteoarthritis. European J Pain. 2018;22(7):1245-1254.

27. Lovibond P. Fear and avoidance: an integrated expectancy model. 2006.

28. Kocic M, Stankovic A, Lazovic M, et al. Influence of fear of movement on total knee arthroplasty outcome. Ann Ital Chir. 2015;86(2):148-155.

29. Herbert MS, Goodin BR, Pero ST IV, et al. Pain hypervigilance is associated with greater clinical pain severity and enhanced experimental pain sensitivity among adults with symptomatic knee osteoarthritis. Annals Behav Med. 2014;48(1):50-60.

30. Thompson DP, Moula K, Woby SR. Are fear of movement, self-efficacy beliefs and fear of falling associated with levels of disability in people with osteoarthritis of the knee? A cross sectional study. Musculoskeletal Care. 2017;15(3):257-262.

31. Sengul Y (Salik), Unver B, Karatosun V, et al. Assessment of pain-related fear in patients with the thrust plate prosthesis (TPP): due to hip fracture and hip osteoarthritis. Arch Gerontol Geriatr. 2011;53(2):e249-e252.

32. Thoma LM. Muscle co-contraction, joint loading, and fear of movement in individuals with articular cartilage defects in the knee. Dissertation Abstracts International: Section B: The Sciences and Engineering. 2019;80(11)

33. Zhang $\mathrm{H}$, Si W, Pi H. Incidence and risk factors related to fear of falling during the first mobilisation after total knee arthroplasty among older patients with knee osteoarthritis: a cross-sectional study. J Clin Nurs. 2021.

34. Spitaels D, Vankrunkelsven P, Desfosses J, et al. Barriers for guideline adherence in knee osteoarthritis care: A qualitative study from the patients' perspective. J Eval Clin Practice. 2017;23(1):165-172.

35. Helminen EE, Sinikallio SH, Valjakka AL, et al. Determinants of pain and functioning in knee osteoarthritis: a one-year prospective study. Clin Rehabil. 2016;30(9):890-900.

36. Odole A, Ekediegwu E, Ekechukwu END, et al. Correlates and predictors of pain intensity and physical function among individuals with chronic knee osteoarthritis in Nigeria. Musculoskelet Sci Pract. 2019;39:150-156.

37. Uritani D, Kasza J, Campbell PK, et al. The association between psychological characteristics and physical activity levels in people with knee osteoarthritis: a cross-sectional analysis. BMC Musculoskeletal Disorders. 2020;21(1):1-7.

38. Gay C, Eschalier B, Levyckyj C, et al. Motivators for and barriers to physical activity in people with knee osteoarthritis: a qualitative study. Joint Bone Spine. 2018;85(4):481-486.

39. Sánchez-Herán Á, Agudo-Carmona D, Ferrer-Peña R, et al. Postural stability in osteoarthritis of the knee and hip: analysis of association with pain catastrophizing and fear-avoidance beliefs. PM R. 2016;8(7):618628.

40. Roelofs J, Sluiter JK, Frings-Dresen MH, et al. Fear of movement and (re)injury in chronic musculoskeletal pain: evidence for an invariant twofactor model of the Tampa Scale for Kinesiophobia across pain diagnoses and Dutch, Swedish, and Canadian samples. Pain. 2007;131(1-2):181190 .

41. Delbaere K, Crombez G, Vanderstraeten G, et al. Fear-related avoidance of activities, falls and physical frailty. a prospective community-based cohort study, Age and Ageing. 2004;33(4):368-373.

42. Holla JFM, Pisters M, Dekker J. Behavioral mechanisms explaining functional decline. In: Dekker $J$, ed. Exercise and Physical Functioning in Osteoarthritis: Medical, Neuromuscular and Behavioral Perspectives. Springer briefs in specialty topics in behavioral medicine. Springer Science + Business Media. 2014:65-85.
43. Stubbs B, Patchay S, Soundy A, et al. The avoidance of activities due to fear of falling contributes to sedentary behavior among communitydwelling older adults with chronic musculoskeletal pain: a multisite observational study. Pain Med. 2014;15(11):1861-1871.

44. Brown OS, Hu L, Demetriou C, Smith TO, et al. The effects of kinesiophobia on outcome following total knee replacement: a systematic review. Arch Orthop Trauma Surg. 2020;140(12):2057-2070.

45. Asmundson GJ, Norton PJ, Norton GR. Beyond pain: the role of fear and avoidance in chronicity. Clin Psychol Rev. 1999;19(1):97-119.

46. Alami S, Boutron I, Desjeux D, et al. Patients' and practitioners' views of knee osteoarthritis and its management: a qualitative interview study. PLoS ONE. 2011;6(5):1-9.

47. Wetherell JL, Bower ES, Johnson K, et al. Integrated exposure therapy and exercise reduces fear of falling and avoidance in older adults: a randomized pilot study. Am J Geriatr Psychiatr. 2018;26(8):849-859.

48. Jönsson T, Eek F, Dell IA, et al. The Better Management of Patients with Osteoarthritis Program: outcomes after evidence-based education and exercise delivered nationwide in Sweden. PLOS ONE. 2019;14(9):1-14.

49. Unver B, Ertekin Ö, Karatosun V. Pain, fear of falling and stair climbing ability in patients with knee osteoarthritis before and after knee replacement: 6 month follow-up study. J Back \& Musculoskeletal Rehabi. 2014;27(1):77-84.

50. Campos DM, Ferreira DL, Gonçalves GH, et al. Effects of aquatic physical exercise on neuropsychological factors in older people: a systematic review. Arch Gerontol Geriatr. 2021;96:104435.

51. Harms A, Heredia-Rizo AM, Moseley GL, et al. A feasibility study of brain-targeted treatment for people with painful knee osteoarthritis in tertiary care. Physiother Theory Pract. 2020 Jan;36(1):142-156.

52. Forss KS, Stjernberg L, Hansson EE, et al. Osteoarthritis and fear of physical activity - the effect of patient education. Cogent Medicine. 2017;4:1.

53. Rosemann T, Wensing M, Joest $\mathrm{K}$, et al. Problems and needs for improving primary care of osteoarthritis patients: the views of patients, general practitioners and practice nurses. BMC Musculoskelet Disord. 2006;2:7-48.

54. Song R, Roberts BL, Lee EO, et al. A randomized study of the effects of T'ai Chi on muscle strength, bone mineral density, and fear of falling in women with osteoarthritis. J Alt Comp Med. 2010;16(3):227-233.

55. Nero H, Dahlberg J, Dahlberg LE. A 6-week web-based osteoarthritis treatment program: Observational quasi-experimental study. $J$ Medical Internet Res. 2017;19(12).

56. Lentz TA, George SZ, Manickas-Hill O, et al. What general and painassociated psychological distress phenotypes exist among patients with hip and knee osteoarthritis? ClinOrthop Relat Res. 2020;478(12):27682783.

57. Vlaeyen JW, Linton SJ. Fear-avoidance and its consequences in chronic musculoskeletal pain: a state of the art. Pain Med. 2000;85(3):317-332.

58. Ikram, M, Ijaz, S, Memon, ML, et al. Prediction of fear factors before total knee replacement: a mixed method analysis on advanced knee osteoarthritis patients. Pakistan Armed Forces Med J. 2020;70(4)988992.

59. Flanigan DC, Everhart JS, Glassman AH. Psychological factors affecting rehabilitation and outcomes following elective orthopaedic surgery. JAAOS. 2015;23(9):563-570.

60. Youngcharoen P, Saraboon Y, Aree US. Factors influencing health status in older people with knee osteoarthritis. Japan J Nurs Sci. 2020;17(1).

61. Romer CM. Stress and coping in older women with osteoarthritis: A qualitative study. Dissertation Abstracts International: Section B: The Sciences and Engineering. 2000;60(9-B):4524. 
62. Vincent KR, Vincent HK. Resistance exercise for knee osteoarthritis. PM\&R. 2012;4(5):S45-S52.

63. An YW. Neuromechanical links between cognition, fear and joint instability. Diss Abstracts Int: Section B: The Sciences and Engineering. 2017;78(4-B(E))

64. Nagai T, Tsurusaki M, Horaguchi T, et al. Verification of psychological factors related to health-related quality of life in elderly knee osteoarthritis: a prospective cohort study. J Orthop Sci. 2020;25(5):868-873.

65. Scopaz KA, Piva SR, Wisniewski S, et al. Relationships of fear, anxiety, and depression with physical function in patients with knee osteoarthritis. Arch Phys Med Rehabil. 2009;90(11):1866-1873.

66. Bhedi JR, Sheth MS, Vyas NJ. Correlation between fear of fall, balance and physical function in people with osteoarthritis of knee joint. Int Arch Integr Med. 2015;2(6):205-209.

67. Miller KA, Osman F, Baier et al. Patient and physician perceptions of knee and hip osteoarthritis care: a qualitative study. Int J Clin Pract. 2020;74(12):e13627.

68. Van Doormaal MCM, Meerhoff GA, Vliet Vlieland TPM, et al. A clinical practice guideline for physical therapy in patients with hip or knee osteoarthritis. Musculoskeletal Care. 2020;18(4):575-595.

69. Vogel M, Riediger C, Krippl M, et al. Negative effect, type D personality, quality of life, and dysfunctional outcomes of total knee arthroplasty. Pain Research \& Management. 2019;2019.
70. Sinikallio SH, Helminen EE, Valjakka AL et al. Multiple psychological factors are associated with poorer functioning in a sample of communitydwelling knee osteoarthritis patients. J Clin Rheumatol: 2014;20(5):261267.

71. Monticone M, Ferrante S, Rocca B, et al. Home-based functional exercises aimed at managing kinesiophobia contribute to improving disability and quality of life of patients undergoing total knee arthroplasty: a randomized controlled trial. Arch Phys Med Rehabil. 2013;94(2):231-239.

72. Tsonga T, Michalopoulou M, Kapetanakis S, et al. Risk factors for fear of falling in elderly patients with severe knee osteoarthritis before and one year after total knee arthroplasty. J Orthop Surg. 2016;24(3):302-306.

73. Gunn AH, Schwartz TA, Arbeeva LS, et al. Fear of movement and associated factors among adults with symptomatic knee osteoarthritis. Arthritis Care Res (Hoboken). 2017;69(12):1826-1833.

74. Nguyen US, Felson DT, Niu J, et al. The impact of knee instability with and without buckling on balance confidence, fear of falling and physical function: the Multicenter Osteoarthritis Study. Osteoarthritis Cartilage. 2014;22(4):527-534.

75. Mat S, Ng CT, Fadzil F, et al. The mediating role of psychological symptoms on falls risk among older adults with osteoarthritis. Clin Interv Aging. 2017; 12:2025-2032. 